\title{
Fair Scheduling of Dynamically Provisioned WDM Connections with Differentiated Signal Quality
}

\author{
Ajmal Muhammad ${ }^{1}$, Cicek Cavdar ${ }^{2}$, Paolo Monti ${ }^{2}$ \\ ${ }^{1}$ Linköping University, Linköping, Sweden, Email: ajmal@isy.liu.se \\ ${ }^{2}$ Royal Institute of Technology (KTH), Stockholm, Sweden, Email: \{cavdar,pmonti\}@kth.se
}

\begin{abstract}
Emerging, on-demand applications (e.g., Interactive video, ultra-high definition TV, backup storage and grid computing) are gaining momentum and are becoming increasingly important. Given the high bandwidth required by these applications, Wavelength Division Multiplexing (WDM) networks are seen as the natural choice for their transport technology. Among the various on-line strategies proposed to provision such services, the ones based on service level agreement (SLA) metrics such as setup delay tolerance and connection holding-time awareness showed a good potential in improving the overall network blocking performance. However, in a scenario where connection requests are grouped in different service classes, the provisioning success rate might be unbalanced towards those connection requests with less stringent requirements, i.e., not all the connection requests are treated in a fair way.

This paper addresses the problem of how to guarantee the signal quality and the fair provisioning of different service classes, where each class corresponds to a specified target of quality of transmission (QoT). With this objective in mind three fair scheduling algorithms are proposed in a dynamic traffic scenario, each one combining in a different way the concept of both set-up delay tolerance and connection holding-time awareness. Proposed solutions are specifically taylored to facilitate the provisioning of the most stringent service class so as to balance the success rate among the different classes. Simulation results confirm that the proposed approaches are able to guarantee a fair treatment reaching up to $99 \%$ in terms of Jain's fairness index, considering the per-class success ratio, without compromising the improvements in terms of overall network blocking probability.

Index Terms-WDM networks, dynamic connection provisioning, connection holding-time, set-up delay tolerance, fairness, scheduling.
\end{abstract}

\section{INTRODUCTION}

Optical network architectures are evolving from traditional opaque wavelength division-multiplexing (WDM) networks toward transparent solutions. In transparent optical networks, also referred to as all-optical networks, an optical circuit (or lightpath) established between two nodes can be routed without requiring any intermediate optical-electrical-optical (OEO) conversion along its route. This approach has its own advantages, namely cost-effectiveness and signal transparency, but also some disadvantages, i.e., the optical signal is affected by the physical characteristics of fibers and network components. In other words phenomena such as crosstalk [1] and spontaneous emission (ASE) noise [2], just to name a few, degrade the quality of the optical signal. As a result, in the absence of regeneration at intermediate nodes, the presence of optical impairments limit the reach of a lightpath [3].
The problem of considering optical impairments while finding, for each lightpath, an appropriate route and a wavelength (i.e., the well known routing and wavelength assignment problem, or RWA) has received a lot of attention from the research community in recent years. The impairment aware routing and wavelength assignment (IA-RWA) takes into account the Quality of Transmission (QoT) of the resulting lightpath during the connection provisioning phase. QoT can be expressed in terms of Bit Error Rate (BER) or, equivalently, in terms of $Q$ factor [2]. Under dynamic traffic scenario, during IA-RWA, connection requests can be blocked for two main reasons. The first one is due to the shortage of wavelength resources, i.e., there are no wavelength routes available between the source and destination node. The other reason is the quality of the optical signal transmitted, i.e., the difficulty to provision a lightpath with the targeted QoT level regardless of the availability of wavelength resources. In other words the BER level of any lightpath between the source and destination node is below the required threshold. The former reason is called wavelength blocking, while the latter is denoted as BER blocking. A comprehensive literature survey on physical layer impairments and on IA-RWA strategies can be found in [4], [5].

In recent years, new, user-controlled, bandwidth intensive applications are becoming increasingly dominant. Applications such as video on demand ( $\mathrm{VoD})$, distribution of ultra-high definition TV (UHDTV), digital cinematic production [6], telecommuting, telemedicine, banking data backup storage, e-science, and grid computing, just to mention a few, are gaining momentum and are considered as attractive and important services for the community. These applications are characterized by different bandwidth and QoT requirements. For example, sensitive backup data (e.g., financial transactions and banking) demands a higher signal quality than that of Gigabit Ethernet [5]. Similarly, media streaming requires a higher signal quality compared to peer-to-peer and World Wide Web (WWW) traffic [7]. Dynamic and on-demand setting up of optical channels [8], [9] is a promising candidate for the provisioning of such applications that not only require high bandwidth but also come with strict service level agreement (SLA) requirements such as a certain level of QoT target. SLA is a contract signed between a customer and a network operator during connection provisioning.

There are several approaches available in the literature addressing the connection provisioning problem for this partic- 
ular set of demanding applications. Among them, the strategies exploiting the time dimension of a connection request have proven to be particularly effective. Set-up delay tolerance $\left(t_{d}\right)$ was defined first in [10] as a customer oriented SLA metric and as the time up to which a connection can be delayed until it is set-up. For example, if a connection request is allowed to wait a pre-defined amount of time before being set-up, namely set-up delay tolerance, then in the absence of resources or in the conditions that a QoT target cannot be met it can be rescheduled for a series of provisioning attempts. In the same way if the holding-time $\left(t_{h}\right)$ of the already established connection requests is known in advance, certain SLA constraints, e.g., reliability for newly established requests, can be relaxed allowing for a more efficient use of network resources and consequently reduction in connection blocking levels. These concepts are known as set-up delay tolerance [10] and holding-time awareness [11], respectively. They have been applied in the context of resource optimization for dynamic lightpath provisioning with [10] and without [12] the assumption of an ideal optical medium. In [13], different service classes were represented by different levels of set-up delay tolerance for different type of services. However in this study we consider the differentiation of service classes in terms of signal quality, where each service class, namely SC-I, SCII, and SC-III, require a different QoT level. When multiple classes of service are considered, the blocking performance of some service classes may not always be as good as the overall network blocking ratio. For example in [13], it was shown that the class of connection requests that have smaller set-up delay tolerance constraint have degraded performance while the total performance was improving. If SC-I is the most demanding class, establishing SC-I connection requests is more challenging than provisioning SC-II and SC-III connection requests. As a result the provisioning success ratio of SC-I is lower than other service classes, because the network resources are mostly assigned to connection requests that do not need such a high transmission quality level. In other words, in a multiclass scenario there is a concern about whether resources are used in a fair way, whereas more demanding classes are blocked more than less demanding classes.

The objective of this paper is to address this fairness problem when set-up delay tolerant and holding-time aware strategies are used to provision bandwidth intensive (i.e., up to at least one wavelength of capacity) connection requests with differentiated classes. In order to promote the higher demanding classes over the lower ones, different priority queuing techniques are used and connection requests are dynamically scheduled within their set-up delay tolerance. For fair scheduling and class differentiation, three provisioning strategies are presented. Each strategy takes advantage in a different way of the combined effect of using the setup delay tolerance and the holding-time awareness concept. There is a trade-off between the overall network performance and fairness. Fairness is not a new concept in the optical networking paradigm. It has been quantified in the general context of circuit switched networks [14] and it is applied to transparent optical network in [15], [16]. In order to quantify the fairness of our fair scheduling strategies with differentiated signal quality, we used Jain's fairness index defined in [14] and we applied it over the provisioning success ratio among three different service classes. Simulation results using a continental core network confirmed the performance improvement of the proposed approaches by also overcoming this trade-off.

The rest of the paper is organized as follows. Section II introduces the general framework for impairment-aware connection provisioning and presents the assumptions for the system. The problem statement and notations are described in section III. The proposed strategies for fair scheduling of on-demand connection provisioning are explained in section IV. The simulation results are shown in section $\mathrm{V}$, and finally some concluding remarks are made in section VI.

\section{IMPAIRMENT AWARE CONNECTION PROVISIONING}

This section describes the general framework for the impairment aware connection provisioning and the Q-factor estimation. Connection requests are assumed to be grouped in three service classes (i.e., SC-I, SC-II, and SC-III), each one requiring a different quality of transmission (QoT) level. It is assumed that the physical components traversed by the optical signal from the source to the destination nodes are: the transmitting laser, the optical crossconnect(s) (OXCs), the optical amplifier(s), the fiber span(s), and the receiver.

The physical layer impairments considered in this work are: optical in-band crosstalk, ASE noise, cross-saturation of erbium-doped fiber amplifier (EDFA), receiver noise, fiber attenuation, and power loss in the optical components.

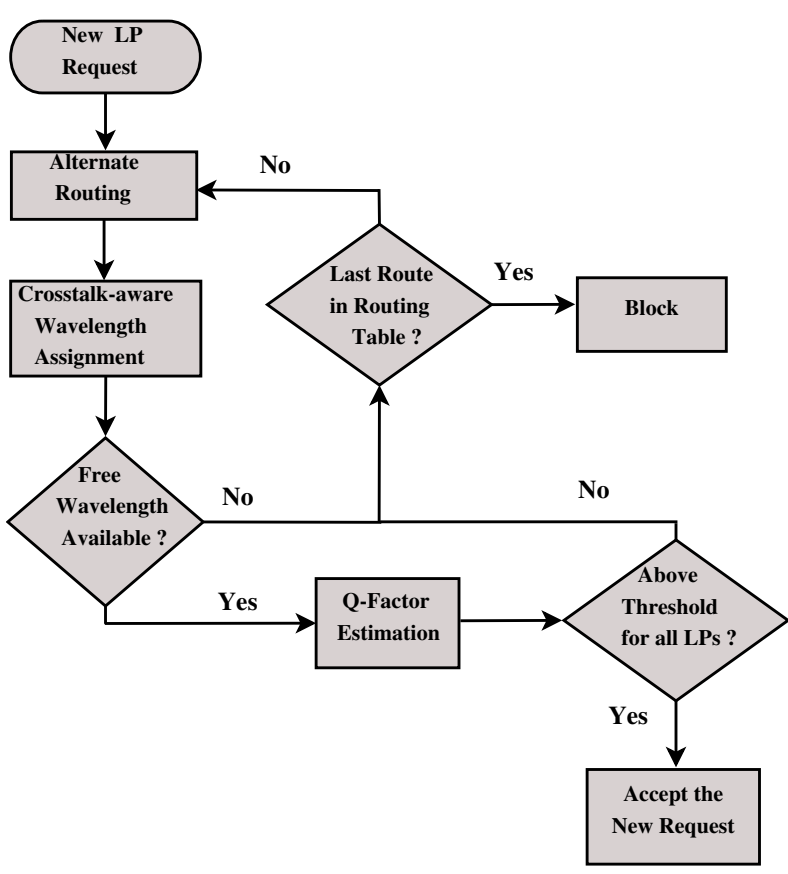

Figure 1. Lightpath admission. 
The out-band crosstalk, originating from connections sharing the same fiber span but using different wavelengths, it is considered negligible in this work, because it can be suppressed using a narrow-band optical filter at the receiver. Similarly, the effect of non linear impairments is not taken into account in this work, by they can be easily included by modifying the transmission impairment model accordingly, i.e., using the one proposed in [17]. Finally it is assumed that Forward Error Correction (FEC) capabilities are not available at the receiver. The in-band crosstalk in our network model is assumed to originate mainly from three components in the OXC: demultiplexer, switching fabric, and multiplexer. The crosstalk between two adjacent channels (adjacent port crosstalk) it is assumed to be dominant over the non-adjacent port crosstalk, i.e., crosstalk originated by signals that are one or more channels away. For the Q-factor estimation we used the model described in [18]-[20] which did not take into account any error correction technique. Mostly applications with tight latency requirements (e.g., interactive communication and streaming data) have less room for error correction, which in turn puts more stringent requirements on the number of acceptable bit errors [21]. In the proposed RWA solution, the routing and wavelength assignment (WA) sub-problems are treated separately. For routing we pre-compute $k$-shortest paths between each source destination pairs. For the WA, we use a QoT-aware scheme called Crosstalk-aware Least-Used (C-LU) algorithm because of its good blocking probability performance [18], [19]. The flowchart of the provisioning framework is shown in Fig.1. The basic concept of C-LU is as follows: Each wavelength available on the considered path is assigned a weighted crosstalk number. This number is a function of the crosstalk experienced by the signal using that particular channel. It is proportional to the number of neighboring channel in use and to the number of components traversed. The higher is the crosstalk experienced by the signal, the higher the crosstalk number for that particular wavelength. The wavelength with the lowest crosstalk number is chosen. If more than one wavelength have the same weighted crosstalk number, then the one is selected which is used on the least number of links in the network.

Establishing a new lightpath might affect negatively the Qfactor of already existing lightpaths. This is due to the inband crosstalk effect. For this reason, after a new lightpath is established, the Q factor of all existing lightpaths sharing any resource with the new one need to be re-checked to make sure that none of them has a QoT level below the required threshold. In other words a new arrival should not disturb the QoT target of an existing connection.

\section{Problem Statement and Notation}

We consider a transparent optical network having non-ideal components in the physical layer with dynamic connection arrivals, each request requiring one wavelength of capacity with a guaranteed QoT level. The fair scheduling problem of dynamically provisioned WDM connections with differentiated signal quality can be formulated as follows: Given: a)
Physical topology of a network represented by a graph $G$ with a set of links $E$ and nodes $V$; maximum number of wavelengths on each link denoted by $W ;$ b) a connection request $R=\left\{s, d, I, t_{a}, t_{h}, t_{d}\right\}$ between a source destination pair $\{s, d\}$ of class $I$, each class corresponding to a signal quality target characterized by a Q-factor threshold $Q_{t h r}$ and with arrival time $t_{a}$, holding time $t_{h}$ and delay tolerance $t_{d}$; c) a set of $k$ pre-computed paths between $\{s, d\}$ denoted by $\pi_{s d, k}$.

Find: A connection $i, C^{i}=\left\{w_{C-L U}^{i}, Q^{i}, p^{i}, t_{s}^{i}\right\}$ is set up on wavelength $w_{C-L U} \in W$ using Crosstalk-aware Least-Used WA scheme with $Q^{i} \geq Q_{t h r}$, on path $p \in \pi_{s d, k}$ with a set-up time $t_{s}$ such that $t_{s} \leq t_{d}-\epsilon$, where $\epsilon$ is a small number.

Objective: Improve the fairness among different classes together with reducing overall network blocking probability by exploiting the set-up delay tolerance and holding-time awareness during provisioning with signal quality differentiation.

\section{Algorithms for Signal Quality Differentiated \\ PROVISIONING WITH SET-UP DELAY TOLERANCE AND HOLDING TIME (DIF-DTHT)}

In this section, we describe a set of strategies for signal quality differentiated provisioning with set-up delay tolerance and holding-time awareness (Dif-DTHT). The objective of these strategies is to promote the connection requests with higher signal quality target in order to enhance the fairness among connection requests belonging to different classes.

\section{A. Dif-DTHT with Backoff (Dif-DTHT-B)}

By using a differentiated backoff strategy, Dif-DTHT-B reschedules connection requests belonging to lower classes to a future time despite the fact they could be provisioned at the time of their arrival. This is done with the intent of enhancing the provisioning chance of new connection requests of class I (SC-I). In general all requests are rescheduled if they can not be set-up at their arrival time $t_{a}$, due to either network layer constraints (wavelength unavailability) or physical layer constraints (signal quality target can not be met). Without reserving any resources, connection requests are rescheduled after the departure of an already provisioned one, if the release of network resources can satisfy the signal quality target of the request within time $t_{d}$ of the connection request. Therefore, the resource allocation phase (RAP) and the provisioning phase (PP) coincide in the time dimension for the Dif-DTHT$\mathrm{B}$ scheme. Moreover, to promote SC-I connection requests, this strategy not only gives them priority over SC-II and SCIII, but also it reschedules SC-II and SC-III requests only after some fraction of their $t_{d}$, even if resources are available. The fraction value $\Delta t_{d}$ used to set the backoff time is different for SC-II and SC-III. In all this study, it is important to note that if a connection request can not be set-up within its setup delay tolerance then the connection is dropped from the rescheduling queue, which is then reordered again.

\section{B. Dif-DTHT with Resource Reservation (Dif-DTHT-RR)}

The Dif-DTHT-RR strategy reserves resources while a connection request is rescheduled. This is done by using the 
information about the holding time of the already provisioned requests. Dif-DTHT-RR computes the set D of all connections which will be released within $t_{d}$. If $\mathrm{D}=0$, or if it is not possible to satisfy the resource or the signal quality requirement of the connection request even after all connections in D have left the network, then the connection request is immediately blocked. Otherwise, Dif-DTHT-RR reserves in advance the resources (wavelength) able to provide the required QoT level. As a result there is a time gap between RAP and PP. In order to promote SC-I requests over the others, resources can only be released and rescheduled if needed by higher class connection requests. Note that reserved resources can be reallocated in favor of a higher class only if the connection is not yet provisioned. After reallocation, the connection belonging to a lower class is rescheduled in the same way as before. This request will be blocked if resources are either not available or can not satisfy the signal quality target within its remaining $t_{d}$.

\section{Dif-DTHT by Mixing two Strategies (Dif-DTHT-Mix)}

This strategy incorporates the salient features of both DifDTHT-B and Dif-DTHT-RR, i.e., it applies the backoff strategy for SC-II and SC-III connections, while it utilizes the concept of advance reservation of network resources only for SC-I requests. The idea behind this strategy is to further improve the accommodation of SC-I connection requests over SC-II and SC-III while still being able to differentiate between SC-II and SC-III via the application of a different backoff time calculation.

\section{NUMERICAL RESUltS}

For performance evaluation of the proposed strategies we custom-built a discrete event-driven simulator and we simulated the proposed strategies on a scaled down version of the NSFnet topology [18], [19]. We assumed to have bidirectional links, where each link has the same number of wavelength, $W=16$, in both directions (Fig.2). It has been shown in [15] that the maximum distance covered by a signal in a transparent optical network (in absence of crosstalk) with a satisfactory Q-factor is less than $1000 \mathrm{~km}$, unless some optimal long-haul link design techniques and components are utilized. This is the reason scaled down versions of the test topologies are used for experiments on transparent optical networks. It is assumed that the network is not equipped with wavelength converters, thus a connection request must use the same wavelength from the source to the destination node. Connections are uniformly distributed among each service class. They are assumed to arrive in the network according to a Poisson process with connection holding-time following an exponential distribution with mean equal to one. The source and the destination of a connection request are uniformly distributed over the set of network nodes. The set-up delay tolerance of each connection has an exponential distribution with mean value equal to 0.1 and the value of $k$ is set to 3 . The value of $\Delta t_{d}$ for SC-II and SC-III is adjusted to 0.5 and 0.7 of the set-up delay tolerance, respectively, in the Dif-DTHT-B and

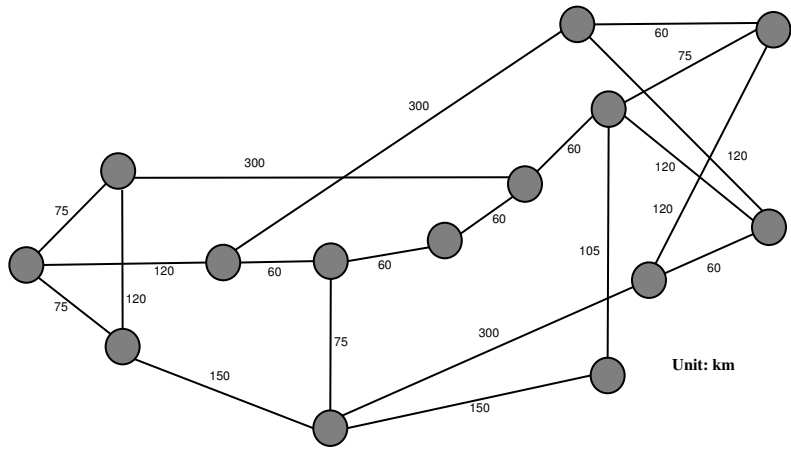

Figure 2. NSF topology scaled down by factor of 10 .

Table I

PARAMETERS AND THEIR VALUES

\begin{tabular}{|c|c|}
\hline Parameter & Value \\
\hline \hline Central wavelength & $1553.6 \mathrm{~nm}$ \\
\hline Channel spacing & $50 \mathrm{GHz}$ \\
\hline Traffic bit rate & $10 \mathrm{Gbps}$ \\
\hline Rx electrical BW $\left(B_{e}\right)$ & $10 \mathrm{GHz}$ \\
\hline Rx optical BW $\left(B_{o}\right)$ & $100 \mathrm{GHz}$ \\
\hline Rx responsivity $(R)$ & $0.95 \mathrm{Amp} / \mathrm{W}$ \\
\hline Rx therm. noise current $\left(I_{t h}\right)$ & $3.8 \times 10^{-12} \mathrm{Amp}$ \\
\hline OXC (de)mux ins. loss & $2 \mathrm{~dB}$ \\
\hline OXC adjacent channel isolation & $25 \mathrm{~dB}$ \\
\hline OXC switch port isolation & $30 \mathrm{~dB}$ \\
\hline Tx source. power & $0 \mathrm{dBm}$ \\
\hline Fiber attn. factor $(\alpha)$ & $0.2 \mathrm{~dB} / \mathrm{km}$ \\
\hline Span length & $75 \mathrm{~km}$ \\
\hline
\end{tabular}

Dif-DTHT-Mix strategies. Table 1 presents the lists physical parameters used in the simulations. More detail about these values can be found in [18] and [19]. Given these values and the assumptions on optical impairments described in Sec II, the receiver-end Q-factor for SC-I, SC-II and SC-III connection requests it is assumed to be equal or greater than 8,7 and 6 , respectively. The network blocking probability (BP), namely, the probability of rejecting a connection requests and the Jain's fairness index value are used to evaluate the performance of our proposed provisioning strategies. The Jain's fairness index, which measures quantitatively the equality of resource allocation, is a number between 0 and 1 defined as:

$$
f_{X}(x)=\frac{\left[\sum_{i=1}^{n} x_{i}\right]^{2}}{n\left[\sum_{i=1}^{n} x_{i}^{2}\right]}
$$

where if $f_{X}(x)$ is equal to 1 for a specific resource $X$ that is shared among $n$ users, then $X$ is considered to be equally shared between all the users [14]. In this paper, network blocking probability can be considered as a quantity to be shared among three different service classes, corresponding in turn to the users. In this way, blocking probability fairness of a service class can be defined as:

$$
f_{S C}(b p)=\frac{\left[\sum_{s c=1}^{n} b p_{s c}\right]^{2}}{n\left[\sum_{s c=1}^{n} b p_{s c}^{2}\right]}
$$




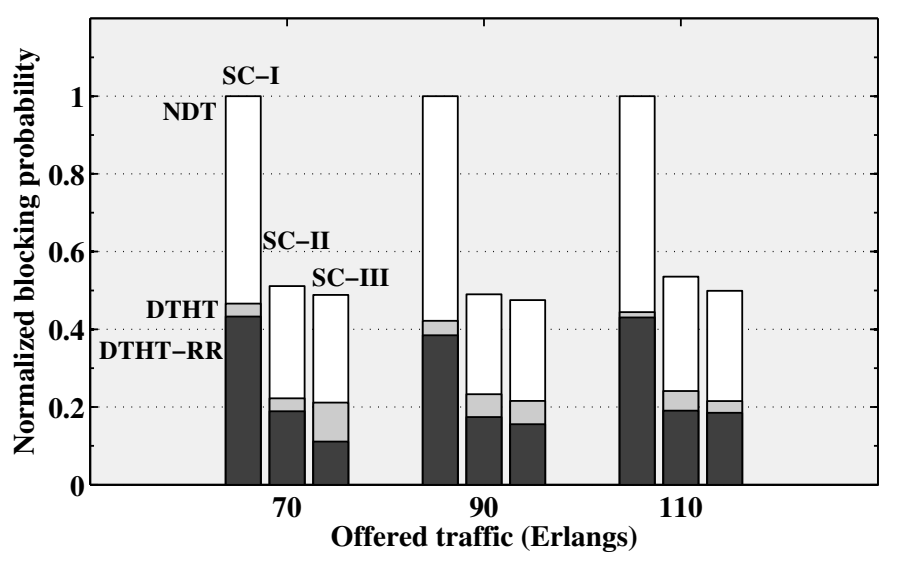

Figure 3. Normalized blocking probability vs. Network load

\section{A. Fairness and Comparison of Different QoT Classes in BP}

In Fig.3, we show the normalized blocking probability in the case of no differentiation among service classes and without giving priority to any of them. The BP is normalized using the SC-I BP value and three different traffic load cases are presented. In order to demonstrate the need for QoT differentiation and fairness, we used a modified version of both the Dif-DTHT-B and the Dif-DTHT-RR strategies. The changes in the strategies are the following: (1) while provisioning different classes with set-up delay tolerance and holding time (DTHT), the backoff strategy is eliminated in Dif-DTHT-B, therefore no differentiation is provided among service classes; (2) in DTHT with resource reservation (DTHT$\mathrm{RR}$ ), once resources are reserved for any class, they can not be reallocated in favor of any higher class. For benchmarking purpose, both DTHT and DTHT-RR are compared with a basic approach, i.e., the impairment-aware provisioning with no delay tolerance (NDT).

Fig. 3 shows that the BP of each class and the total network BP are reduced significantly when a set-up-delay-tolerancebased and a holding time aware approach is used, i.e., an improvement of $56 \%$ and $63 \%$ in the total network BP for DTHT and DTHT-RR strategies, respectively. However, the BP of SC-I is $48 \%$ higher than SC-II for NDT, and this difference becomes more significant when the DTHT and DTHTRR approaches are used, i.e., 50\% and 53\%, respectively. Fig.3 shows some sort of fairness strategies among different service classes are required despite the fact that set-up delay tolerance and holding time awareness can help to reduce the BP.

\section{B. Blocking Probability Comparison Under QoT Differentia- tion}

Our proposed strategies aim at providing fairness among different service classes. Dif-DTHT-B, Dif-DTHT-RR and DifDTHT-Mix are compared in terms of total BP in Fig.4(a) and in terms of SC-I BP in Fig.4(b). In Fig.4(a), it can be seen that Dif-DTHT-RR algorithm, by reserving resources for all

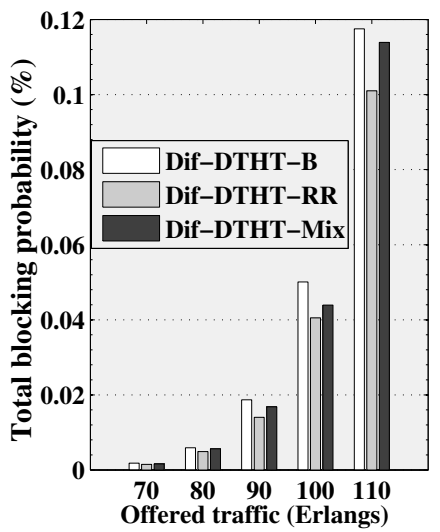

(a)

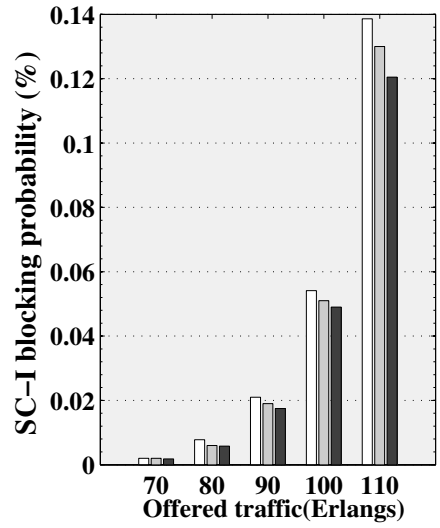

(b)
Figure 4. Blocking probability vs. Network load (a) Total (b) SC-I

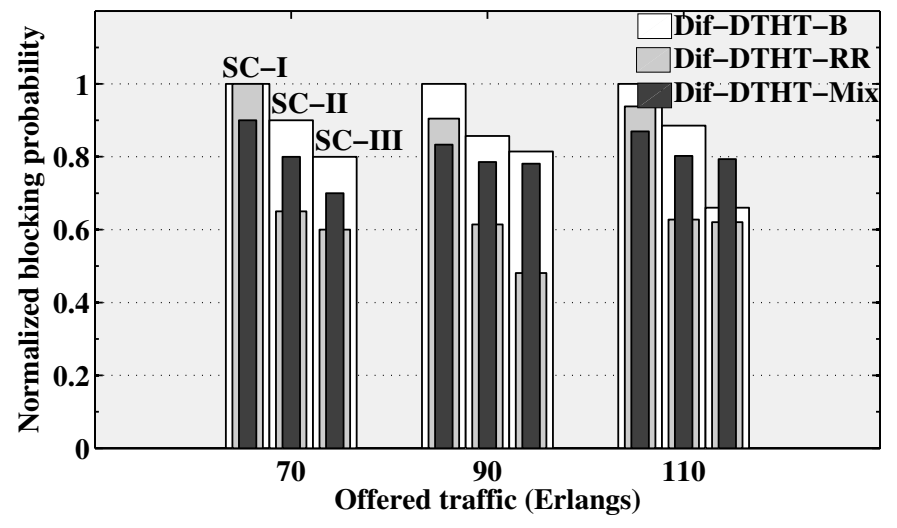

Figure 5. Normalized blocking probability vs. Network load

three classes, outperforms the other approaches in terms of total BP. When it comes to SC-I BP, Dif-DTHT-Mix gives the best $\mathrm{BP}$ reduction since it promotes SC-I over the other two classes by using a mixture of both backoff (for SC-II and SC-III) and resource reservation (only for SC-I) (Fig.4(b)). In order to show a clear picture of fairness among different service classes, the normalized value of BP is shown in Fig.5 for three different loads. In this figure, the value of $\mathrm{BP}$ for SC-II and SC-III is normalized to the value of BP of SC-I for all load levels. Among the three different proposed priority scheduling strategies, the most promising approach in terms of fairness is Dif-DTHT-Mix. In very low load when the overall BP is very low, both Dif-DTHT-B and Dif-DTHT-RR perform equally in terms of SC-I BP, while Dif-DTHT-Mix is $11 \%$ lower. For medium and high load, Dif-DTHT-RR performs better than Dif-DTHT-B and Dif-DTHT-Mix outperforms the two other approaches in terms of SC-I BP reduction. For low and medium load, the value of BP for SC-II and SC-III is higher with Dif-DTHT-Mix when compared to the Dif-DTHTRR case and it is still better than the BP value of Dif-DTHT-B. In order to decrease the BP of SC-I, Dif-DTHT-Mix sacrifices 
Table II

FAIRNESS IN TERMS OF SERVICE CLASS BP

\begin{tabular}{|c|l|l|l|l|l|}
\hline \multirow{2}{*}{ Provisioning strategy } & \multicolumn{5}{|c|}{ Network load (Erlangs) } \\
\cline { 2 - 6 } & 70 & 80 & 90 & 100 & 110 \\
\hline NDT & 0.87 & 0.89 & 0.87 & 0.88 & 0.88 \\
\hline DTHT & 0.85 & 0.88 & 0.86 & 0.88 & 0.87 \\
\hline DTHT-RR & 0.76 & 0.86 & 0.82 & 0.81 & 0.82 \\
\hline Dif-DTHT-B & 0.98 & 0.93 & 0.98 & 0.98 & 0.96 \\
\hline Dif-DTHT-RR & 0.93 & 0.95 & 0.92 & 0.95 & 0.95 \\
\hline Dif-DTHT-Mix & 0.98 & 0.98 & 0.98 & 0.99 & 0.98 \\
\hline
\end{tabular}

\section{the BP of SC-II and SC-III.}

\section{Fairness Index for different strategies}

The Jain's fairness index values for the different provisioning strategies at different network loads is reported in Table II. These values confirm the same trend witnessed so far, i.e. exploiting the set-up delay tolerance without giving special attention to SC-I connection requests affects the network fairness. Dif-DTHT-Mix presents the highest fairness index value among all network loads.

\section{CONCLUSION}

In this paper, we proposed three different algorithms for fair scheduling of dynamically provisioned WDM connections with differentiated signal quality. For the QoT measurement we used the Q-factor of the signals in the network. First we show that exploiting both the holding-time knowledge and the set-up delay tolerance, one can improve the overall BP of a network, i.e., if the QoT target of a connection can not be met at a specific time, it might be met in the future when an existing connection departs from the system. However, with such a strategy, the service class which requires relatively low QoT (SC-III) has more chance to be successfully provisioned when compared to the other (more stringent) classes.

In order to balance the provisioning success rate among all service classes and to improve the fairness of the network, we proposed three different solutions. The performance of these strategies varies in terms of total network BP and BP of the most stringent service class. This will enable the network operator to select a strategy based on up to which level the blocking of the most stringent service class need to be improved. Moreover, among the proposed algorithms, DifDTHT-Mix demonstrates better performance because it not only achieves a high fairness index value, but it also exhibits better performance in terms of total network BP compared to Dif-DTHT-B. It is likely that fair scheduling strategies will be important in the near future when customer oriented SLA metrics, i.e., set-up delay tolerance and holding time awareness will be exploited in networks having services with different QoT requirements.

\section{ACKNOWLEDGMENT}

The work described in this paper was carried out with the support of "Security in All-optical network" project, funded by VINNOVA (The Swedish Governmental Agency for Innovation Systems).

\section{REFERENCES}

[1] I. T. Monroy, Crosstalk in WDM communication networks. Khuwer Academic Publishers, 2002.

[2] G. P. Agrawal, Fiber-optic communication systems. John Wiley \& Sons, Inc., 2002.

[3] E. Goldstein and L. Eskildsen, "Scaling limitations in transparent optical networks due to low-level crosstalk," Photonics Technology Letters, IEEE, vol. 7, no. 1, pp. 93 -94, jan 1995.

[4] S. Azodolmolky, M. Klinkowski, E. Marin, D. Careglio, J. S. Pareta, and I. Tomkos, "A survey on physical layer impairments aware routing and wavelength assignment algorithms in optical networks," Comput. Netw., vol. 53, pp. 926-944, May 2009.

[5] C. Saradhi and S. Subramaniam, "Physical layer impairment aware routing (pliar) in wdm optical networks: issues and challenges," Соттиnications Surveys Tutorials, IEEE, vol. 11, no. 4, pp. $109-130$, quarter 2009.

[6] D. Shirai, T. Kawano, T. Fujii, K. Kaneko, N. Ohta, S. Ono, S. Arai, and T. Ogoshi, "Real time switching and streaming transmission of uncompressed 4k motion pictures," Future Gener. Comput. Syst., vol. 25, pp. 192-197, February 2009.

[7] “Tramms ip traffic reports no.1 \& 3, april \& june 2008," Tech. Rep., traffic measuremt and models in multi-service network project, celtic project,. [Online]. Available: http://projects.celtic-initiative.org/tramms/.

[8] D. Simeonidou, R. Nejabati, G. Zervas, D. Klonidis, A. Tzanakaki, and M. O'Mahony, "Dynamic optical-network architectures and technologies for existing and emerging grid services," Lightwave Technology, Journal of, vol. 23, no. 10, pp. 3347 - 3357, oct. 2005.

[9] B. Mukherjee, "Architecture, control, and management of optical switching networks," in Photonics in Switching, 2007, aug. 2007, pp. 43 -44.

[10] C. Cavdar, M. Tornatore, F. Buzluca, and B. Mukherjee, "Shared-path protection with delay tolerance (sdt) in optical wdm mesh networks," Lightwave Technology, Journal of, vol. 28, no. 14, pp. $2068-2076$, july15 2010.

[11] M. Tornatore, C. Ou, J. Zhang, A. Pattavina, and B. Mukherjee, "Photo: an efficient shared-path-protection strategy based on connection-holdingtime awareness," Lightwave Technology, Journal of, vol. 23, no. 10, pp. $3138-3146$, oct. 2005.

[12] A. Muhammad, R. Forchheimer, and L. Wosinska, "Impairment-aware dynamic provisioning in wdm networks with set-up delay tolerance and holding-time awareness," in 2011. ICON 2011. Proceedings. 17th International Conference on Networks, Dec. 2011.

[13] A. Muhammad, C. Cavdar, L. Wosinska, and R. Forchheimer, "Effect of delay tolerance in wdm network with differentiated services," in Optical Fiber Communication - incudes post deadline papers, 2011. OFC 2011. Conference on, march 2011, pp. 1 -3.

[14] "A quantitative measure of fairness and discrimination for resource allocation in shared computer systems," Tech. Rep., sep. 1984, dEC Research Report TR-301.

[15] Y. Pointurier, M. Brandt-Pearce, T. Deng, and S. Subramaniam, "Fair qos-aware adaptive routing and wavelength assignment in all-optical networks," in Communications, 2006. ICC '06. IEEE International Conference on, vol. 6, june 2006, pp. $2433-2438$.

[16] Y. Pointurier, M. Brandt, T. Deng, and S. Subramaniam, "Fair routing and wavelength assignment in all-optical networks," in Optical Fiber Communication Conference, OFC 2006, march 2006, pp. 1-3.

[17] A. Jirattigalachote, P. Monti, L. Wosinska, K. Katrinis, and A. Tzanakaki, "Icbr-diff: an impairment constraint based routing strategy with quality of signal differentiation," Journal of Networks, vol. 5, no. $11,2010$.

[18] S. Deng, T. Subramaniam, "Qos-friendly wavelength assignment in dynamic wavelength-routed optical networks," Photonic Network Communications, SpringerLink, vol. 10, pp. 5-22, 2005.

[19] T. Deng, S. Subramaniam, and J. Xu, "Crosstalk-aware wavelength assignment in dynamic wavelength-routed optical networks," in Broadband Networks, 2004. BroadNets 2004. Proceedings. First International Conference on, oct. 2004, p. 140.

[20] R. Ramaswami and K. N. Sivaraian, Optical Networks - A Pratical Perspective. CA: Morgan Kaufmann, 2010.

[21] Tech. Rep., bit-error rate (BER) for high-speed serial data communication,. [Online]. Available: http://www.analogzone.com/nett1003.pdf 